\title{
Raynaud's Phenomenon in a Patient with a High Eosinophil Count and Hypocomplementemia. A Case Report and Literature Review
}

\author{
Ahmad Al-Shami, Naser Al Hadhood \\ Department of Rheumatology, Farwaniya Hospital, Kuwait City, Kuwait \\ Email: drahmadalshami@yahoo.com,nalhadhood@gmail.com
}

How to cite this paper: Al-Shami, A. and Al Hadhood, N. (2022) Raynaud's Phenomenon in a Patient with a High Eosinophil Count and Hypocomplementemia. A Case Report and Literature Review. Open Journal of Rheumatology and Autoimmune Diseases, 12, 46-55.

https://doi.org/10.4236/ojra.2022.121006

Received: November 5, 2021

Accepted: February 25, 2022

Published: February 28, 2022

Copyright $\odot 2022$ by author(s) and Scientific Research Publishing Inc. This work is licensed under the Creative Commons Attribution International License (CC BY 4.0).

http://creativecommons.org/licenses/by/4.0/

\begin{abstract}
This report describes clinical findings of a 28 -year old female patient presented with non-healing digit ulcer and Raynaud's phenomenon. Upon investigation was found to have high eosinophil count alongside hypocomplementemia. This patient was diagnosed with diffuse cutaneous systemic sclerosis and was started on therapy; her other laboratory findings were attributed to a coinciding helminthic infection. This case suggests the possibility of having two different diagnoses presenting at once causing a clinical dilemma.
\end{abstract}

\section{Keywords}

Systemic Sclerosis, Eosinophilia, Hypocomplementemia

\section{Case Report}

A 28-year-old previously healthy female of East Asian decent was admitted due to hypoglycemia. The patient initially sought medical attention for a non-healing finger ulcer at the tip of the left index finger, at the primary Healthcare Centre she was found to be hypoglycemic and referred to the hospital for further workup. Upon arrival to the hospital the patient complained of dyspnea and showed decreased saturation on the pulse oximetry; in addition, she also added a history of palpitations on and off. The patient was suspected to have a pulmonary embolism and was taken for a Computed Tomography Pulmonary Angiogram (CTPA) to rule out pulmonary embolism. Her CTPA did not show pulmonary embolism yet showed bilateral basal ground-glass opacities furthermore she was 
found to be hypoglycemic on three occasions. Upon further questioning she was found to be having a history of discoloration in her hands which was more evident upon using the freezer, the refrigerator or at cold weather.

This feature of hand discolouration has been there for at least two months; which indicates true Raynaud's phenomenon. Upon further questioning the patient claimed to have dyspnea on exertion for at least one year, this was associated with generalized fatigue; No history of gastroesophageal reflux disease (GERD), abdominal pain, rash, oral ulcers, eyes symptoms, hair loss, arthritis, neurological symptoms, constitutional symptoms, Deep vein thrombosis nor miscarriage. The hypoglycemia was managed accordingly, and the appropriate workup was sent.

On examination, her vital signs showed a blood pressure of 100/66 $\mathrm{mmHg}$, Heart rate of 70 beats per minute and oxygen saturation was $68 \%$ from her finger yet when taken from the ear lobe was $99 \%$ on room air; the respiratory rate was 12 breaths per minute and she looked comfortable in bed. The patient shows signs of low mood. She had evidence of acne scarring in her face. Upon examining the patient mouth there was no restriction in the mouth opening nor thickened frenulum and also no skin tightening in her face. There was no evidence of dryness in eyes nor mouth; no mouth ulcers were present. Hand examination shows active Raynaud's phenomenon. There was evidence of skin tightening of fingers extending proximally to the metacarpophalangeal joints (MCPs) reaching up to the mid forearms; with was an ulcer in the left index finger. There were scarring in the tip of fingers, yet no nail changes were present. There was no evidence of sclerodactyly telangiectasia nor rash, See Figure 1 and Figure 2. The

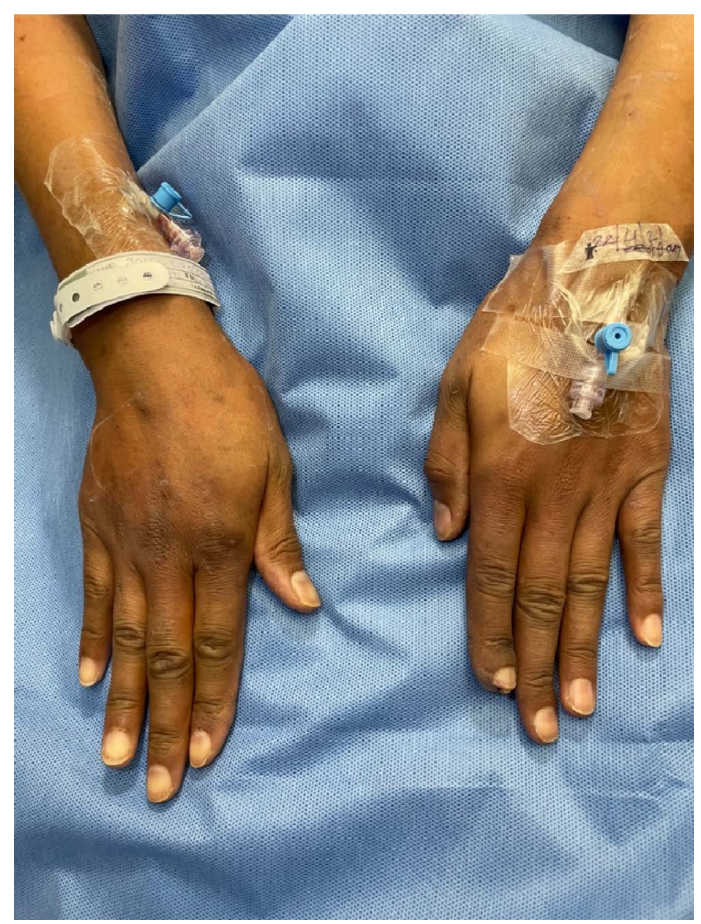

Figure 1. Displaying the patient hands. 


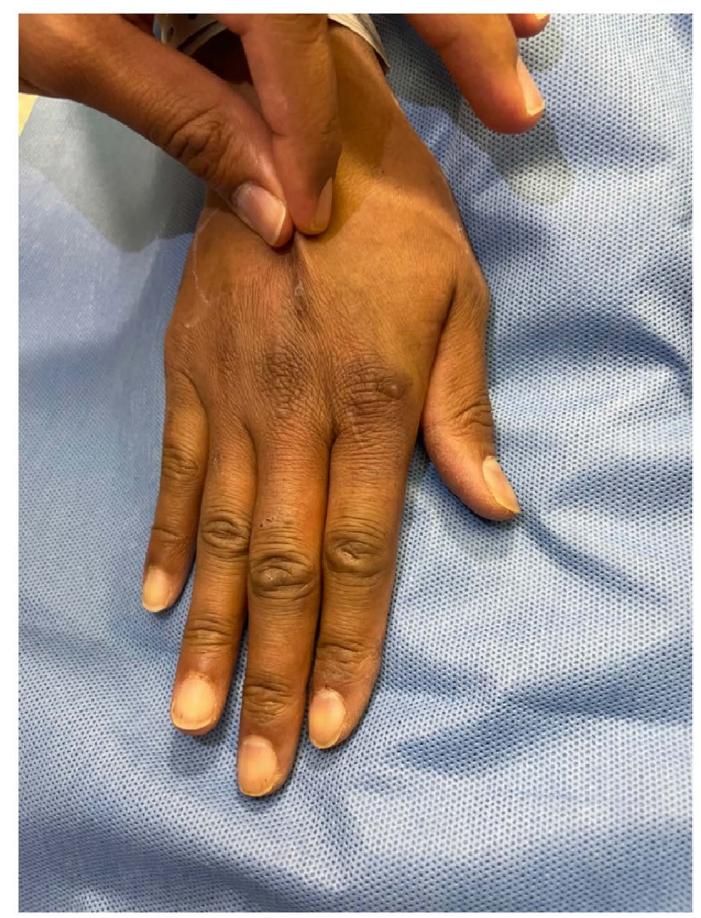

Figure 2. Evidence of skin tightening proximal to MCP.

joint examination shows no synovitis nor calcinosis. Furthermore, the feet examination was positive for tight skin of toes extending proximal to the metatarsophalangeal joints (MTPs) up to mid-shins. Chest examination reveals fine crepitation bilaterally at the lung bases. Cardiovascular examination shows regular first and second heart sound, yet the second heart sound had a loud pulmonary component. The rest of systemic examination was nonsignificant. Capillaroscopy showed microaneurysm in the fourth right nailbed.

The blood test has shown a white-cell count was 7000 per cubic millimeter, with $28 \%$ Neutrophils, $21 \%$ Lymphocytes, $8 \%$ Monocytes, and $42 \%$ Eosinophils. The absolute eosinophilic count was $3 \times 10^{9} / \mathrm{L}$ (normal range $0.1-0.5 \times 10^{9} / \mathrm{L}$ ). The hemoglobin level was 16.5 grams per deciliter with a mean corpuscular volume 95 femtoliters and the platelet count was 142,000 per cubic millimeter. Peripheral blood smear showed eosinophilia, reactive lymphocytes left shift to band form, platelets decreased with no clumps. The partial thromboplastin time was 33.5 seconds (normal range 22.0 to 36.0 ), the international normalized ration (INR) was 1.21 (normal range, 0.9 to 1.1 ). The serum level of sodium was $133 \mathrm{mmol}$ per litre, potassium level $3.9 \mathrm{mmol} / \mathrm{L}$ and chloride was $104 \mathrm{mmol} / \mathrm{L}$. blood urea nitrogen level $4.2 \mathrm{~g}$ per deciliter, and creatinine level $53 \mathrm{mmol} / \mathrm{L}$. Serum Albumin was 26 grams per litre (normal range, 35 - 48). The level of aspartate aminotransferase was $104 \mathrm{U}$ per litre (normal range, 10 to 50), the level of alanine aminotransferase $66 \mathrm{U}$ per litre (normal range, 10 to 50), and the level of alkaline phosphatase $72 \mathrm{U}$ per litre (normal range, 40 - 130) Total Bilirubin was $16.5 \mathrm{mmol} / \mathrm{L}$ (normal range 3 - $20 \mathrm{mmol} / \mathrm{L}$ ) Serum Amylase, Lipase, Creatinine Kinase were within normal limit. Morning cortisol $1426 \mathrm{mmol} / \mathrm{L}$ (normal range 
180 - 625). Anti-Insulin Antibody was negative. Hemoglobin A1C was 5.6\%, Urine microscopy was normal with no casts, white cells, red cells nor protein. C-reactive protein was normal with slight elevation of Erythrocyte sedimentation rate to $42 \mathrm{~mm} / \mathrm{hr}$. complement levels are as follows, complement 3 (C3) was $0.84 \mathrm{~g} / \mathrm{L}$ (normal range 0.88 - 2) and complement 4 (C4) was $0.11 \mathrm{~g} / \mathrm{L}$ (normal range 0.16 - 0.47). Immunology panel is seen in Table 1 and Table 2.

The patient underwent a Chest X-Ray and a CT scan of the Chest, Figure 3 and Figure 4. She underwent a Gastroduodenoscopy which was done part of the workup of SSc Figure 5. The Gastroduodenoscopy result showed a normal esophagus with normal mucosa and no hiatus hernia. The stomach showed evidence of moderate patchy erythema. The duodenum had evidence of erythema throughout with a mobile roundworm. Figure 6 shows the hookworm taken from the Gastroduodenoscopy.

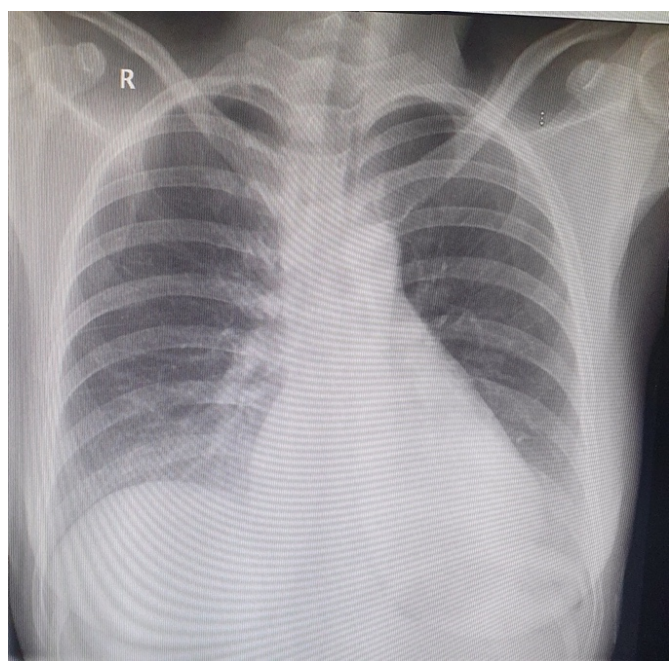

Figure 3. Chest X-Ray.

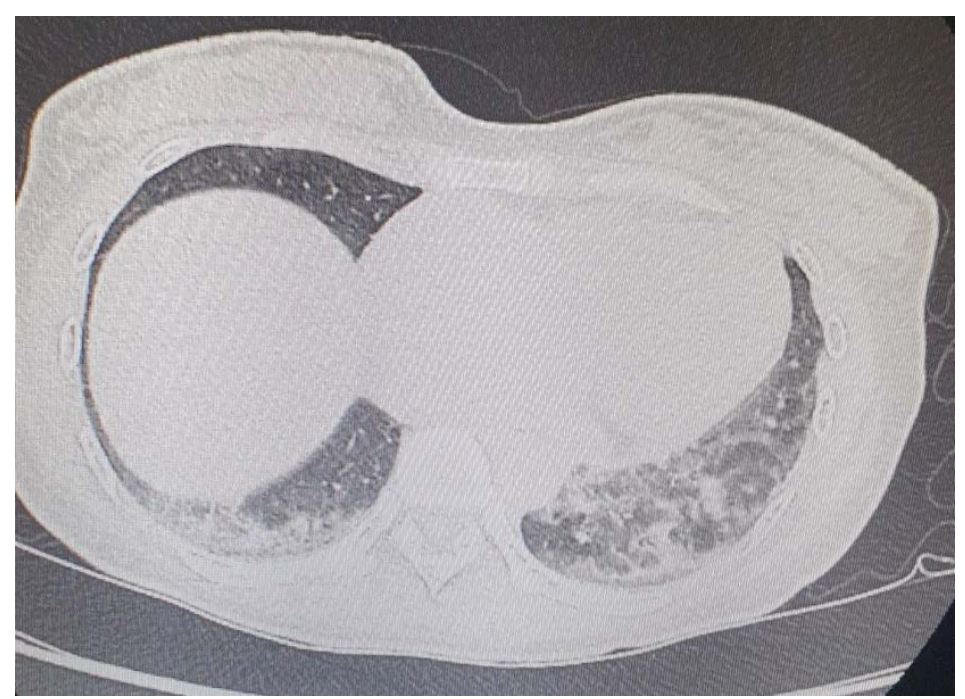

Figure 4. CT Chest: Demonstrating basal ground glass opacities noted in the lingula and lower lobes bilaterally. 


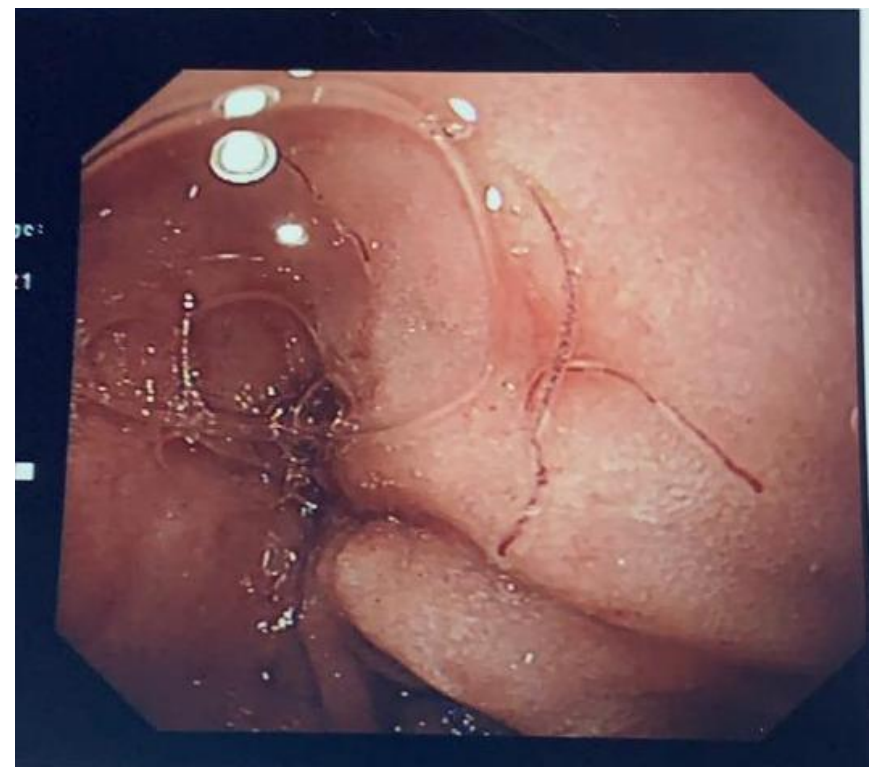

Figure 5. Gastroduodenoscopy findings of the hookworm. Hyperemic mucosa and numerous mobile roundworms in the Duodenum.

Table 1. Immunology panel.

\begin{tabular}{ccc}
\hline Antibody Test & Result & Reference \\
\hline Anti-double stranded & $<1 / 10$ & $<1 / 10$ \\
DNA Antibody (dsDNA) & & \\
Anti Nuclear Antibody & $1 / 640$ & $<1 / 80$ \\
(ANA) & Speckled and homogenous cytoplasmic fibers & \\
\hline
\end{tabular}

Table 2. Extractable nuclear antigen (ENA) profile.

\begin{tabular}{cl}
\hline Antibody Test & Result \\
\hline Anti-Smith (Sm) & Negative \\
Anti RNP Ribonucleoprotein & Positive \\
Anti Jo-1 & Negative \\
Anti SCL 70 Topoisomerase & Positive \\
Anti SSA (Ro) & Negative \\
Anti SSB (La) & Negative \\
Anti-Histone & Negative \\
Anti sm/RNP & Positive \\
Anti Ku & Negative \\
Anti Mi-2 & Negative \\
Anti SRP & Negative \\
Anti-Ribosome PO & Negative \\
Anti PM-Scl 100 & Negative \\
& \\
\hline &
\end{tabular}




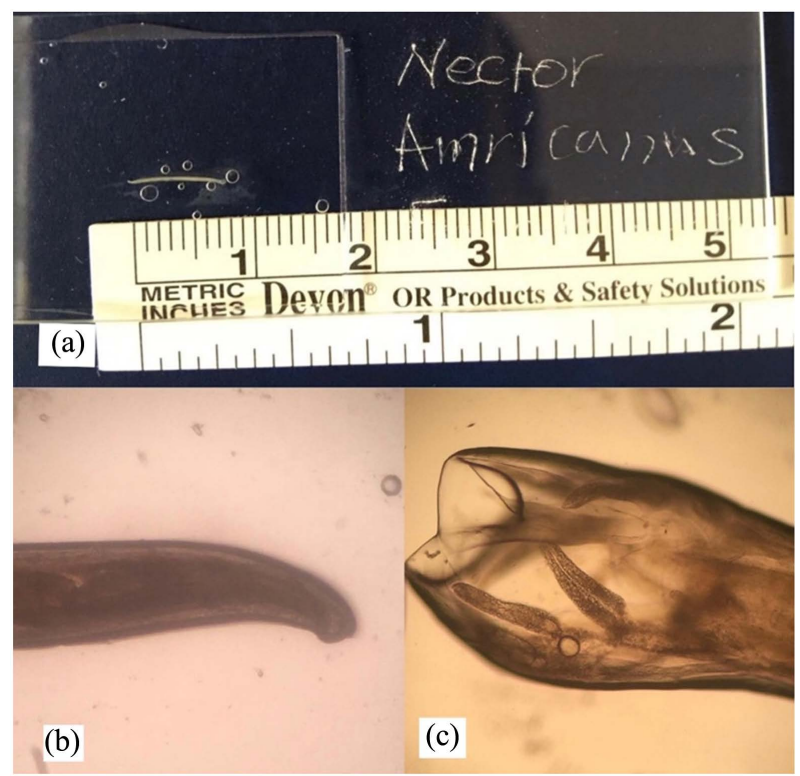

Figure 6. Showing the hookworm (a) Necator americanus next to a ruler showing the length of $8 \mathrm{~mm}$; (b) shows the Tail end of the hook-worm; (c) showing the mouth of the organism.

A diagnosis of Diffuse Cutaneous Systemic Sclerosis (DsSSc) was made the patient was started on Amlodipine, Aspirin and Omeprazole accordingly. Regarding the Helminthic infection she was started on Praziquantel and Mebendazole with improvement seen as the patient's eosinophil count decreased from 3 to $1.9 \times 10^{9} / \mathrm{L}$ (normal range $0.1-0.5 \times 10^{9} / \mathrm{L}$ ). The patient reported improvement with regards to the Raynaud's phenomenon. With this improvement, she was discharged, and started on Mycophenolic acid for her interstitial lung disease and given follow-up appointment.

\section{Discussion}

As the patient presented with hypoglycemia on three occasions accompanied by a borderline blood pressure, fatiguability, skin darkening, and eosinophilia; the first differential diagnosis would be adrenal insuffiency, yet morning cortisol was normal therefore it was excluded. As in Raynaud's phenomenon and ground glass opacity in the lung one would think of Systemic sclerosis, Systemic lupus erythematosus (SLE), Mixed connective tissue disease, Rheumatoid arthritis, or Sjogren syndrome. With the patient's typical features of skin tightening, systemic sclerosis (SSc) was kept as the most likely diagnosis. Hypocomplementemia accompanied with eosinophilia pointed to a diagnosis other than SSc. If SLE was part of overlap in this case; this hematological profile of high eosinophil count does not indicate that diagnosis. Given the fact that the patient is of east Asian descent, Helminthic infection is to be ruled out in this context of hypocomplementemia and eosinophilia. Mild elevation of transaminase, prolonged prothrombin time, recurrent hypoglycemia, slight increments in bilirubin, mainly indirect bilirubin, and low albumin level all support the diagnosis of chronic liver 
disease. However, as the ultrasound of the liver showed a normal liver parenchyma. The previously mentioned abnormal liver function tests can be attributed to helminthic infection. The patient's upper gastrointestinal endoscopy showed worm infestation. Therefore, a diagnosis of Systemic sclerosis was made alongside a helminthic infection.

Scleroderma is a connective tissue disease characterized by skin thickening and tightening. It is divided into two main form of localized and systemic. The localized form also referred to by scleroderma, is further classified as localized, or morphea, and generalized. There are further classifications of localized scleroderma which involves the skin only. The other major form of scleroderma is referred to systemic sclerosis [1]. Systemic sclerosis (SSc), is a complex multiorgan disease characterized by autoimmunity, inflammation, vasculopathy, and progressive fibrosis of the skin and visceral organs. In general, the extent of skin involvement and its rate of progression reflect the severity of visceral organ complications [2] [3]. Furthermore, SSc is divided into three subtypes based on the extent of skin involvement: limited cutaneous systemic sclerosis (LcSSc), diffuse cutaneous systemic sclerosis (DcSSc), and systemic sclerosis sine scleroderma. The latter being with internal organ involvement alongside is positive antibodies and absent characteristic skin findings of scleroderma. LcSSc is associated with internal organs fibrosis and pulmonary artery hypertension; it is also characterized by CREST syndrome, which stands for calcinosis, Raynaud's phenomenon, esophageal dysmotility, sclerodactyly and telangiectasia. DcSSc is characterized by diffuse skin involvement and more commonly associated with interstitial lung disease and scleroderma renal crisis.

Systemic Sclerosis can be difficult to differentiate from localized scleroderma and eosinophilic fasciitis since all three-share cutaneous fibrosis. The presence of symmetric skin thickening in the fingers, hand and forearm are hallmark of systemic sclerosis alongside systemic involvement [4]. The diagnosis of systemic sclerosis is made by history and clinical examination. The presence of specific clinical findings, alongside specific autoantibodies is essential in the establishment of diagnosis of Systemic sclerosis. The use of imaging is such as High resolution computed tomography (HRCT) and Echocardiography is also important in the diagnosis process and follows up. In 2013, a revised classification criteria-the American College of Rheumatology (ACR) - European League Against Rheumatism (EULAR) criteria-were proposed to address some of the difficulties in classification and diagnosing scleroderma Table 3 [5].

The patient is diagnosed with DcSSc, this is based on the timing of Raynaud's phenomenon in relation to other symptoms. The Raynaud's phenomenon which was present in the case was described to have started around two months from the first presentation to the hospital. The patient had Raynaud's phenomenon which is a symptom of reversible peripheral arteriolar vasoconstriction, in response to cold or stress [6]. In this patient, the color changes of the fingertips on both hands when they are in cold will turn sometimes bluish. The progressive nature of the patient's shortness of breath over the span of 12 months alongside 
Table 3. The ACR-EULAR criteria for the classification of systemic sclerosis.

\begin{tabular}{|c|c|c|}
\hline Manifestations & Additional Manifestations & Weight/Score \\
\hline $\begin{array}{l}\text { Skin thickening of fingers } \\
\text { of both hands extending } \\
\text { proximal to MCPs }\end{array}$ & - & 9 \\
\hline & Puffy fingers & 2 \\
\hline $\begin{array}{l}\text { Skin thickening of fingers } \\
\text { (count higher score only) }\end{array}$ & $\begin{array}{l}\text { Sclerodactyly of fingers distal to } \\
\text { the MCPs but proximal to PIPs }\end{array}$ & 4 \\
\hline \multirow{2}{*}{ Fingertip lesions } & Digital tip ulcers & 2 \\
\hline & Fingertip scars & 3 \\
\hline Telangiectasia & - & 2 \\
\hline Abnormal nailfold capillaries & - & 2 \\
\hline $\begin{array}{l}\text { Pulmonary hypertension } \\
\text { and/or interstitial lung }\end{array}$ & Pulmonary hypertension & 2 \\
\hline disease (maximum score is 2 ) & Interstitial lung disease & 2 \\
\hline \multirow[t]{2}{*}{ Raynaud's phenomenon } & - & 3 \\
\hline & Anticentromere & 3 \\
\hline \multirow[t]{2}{*}{$\begin{array}{l}\text { SSc-related autoantibodies } \\
\text { (maximum score is } 3 \text { ) }\end{array}$} & Anti-Scl 70 (Antitopoisomerase-1) & 3 \\
\hline & Anti-RNA polymerase III & 3 \\
\hline
\end{tabular}

The patient discussed in this case had a score of 16 , hence the diagnosis of SSc was made

the finding of Interstitial lung disease and the presence of the Scl 70 Antibody, direct toward the most likely diagnosis of DcSSc.

Eosinophilia refers to elevation in the eosinophil count more than $0.5 \times 10^{9} / \mathrm{L}$ (normal range $0.1-0.5 \times 10^{9} / \mathrm{L}$ ). Elevated Absolute Eosinophil count more than $1.5 \times 10^{9} / \mathrm{L}$ is considered significant and should be investigated further. Eosinophilia is most frequently caused by allergic diseases, parasites, skin diseases or drugs. Sometimes no underlying cause is found, if the eosinophil count is elevated $\left(>1.5 \times 10^{9} / \mathrm{L}\right)$ for over six months and associated with tissue damage then hypereosinophilic syndrome is the diagnosis [7]. Such patients have involvement of the heart valves, central nervous system, skin and lungs with steroids and cytotoxic drugs as the treatment options. Table 4 demonstrates the different cause of increased blood eosinophils. Figure 7 is the result of the patient's peripheral blood smear showing eosinophilia.

Parasitic diseases caused by worms are also referred to as Helminths, they are macroparasites seen by the naked eyes [8]. Helminths are used to refer to worms that are intestinal parasites. Hookworm is among the most important soil-transmitted helminths for humans worldwide [9]. Such previously mentioned infections by helminths are very common and present with blood loss, iron-deficiency anemia, and other anemia associated symptoms and signs. Around the world human hookworm includes two species, Necator americanus 


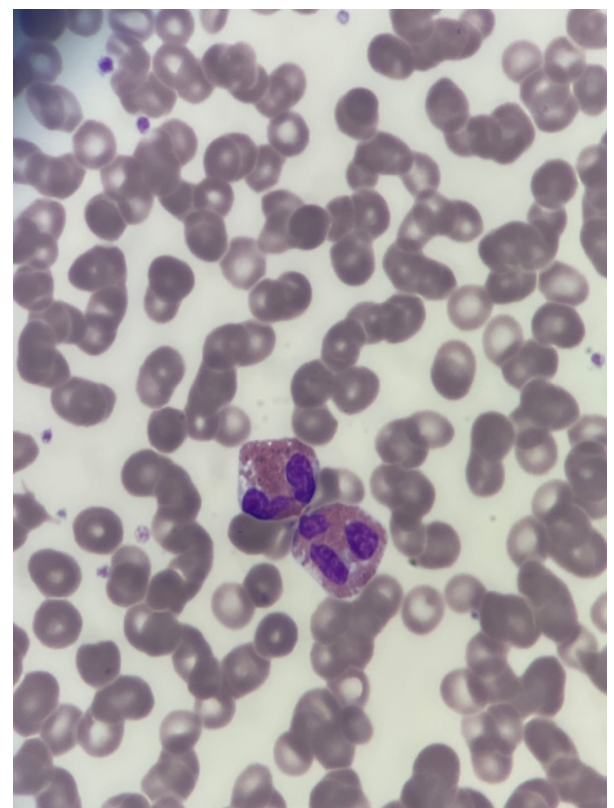

Figure 7. The result of peripheral blood smear showing the evidence of eosinophilia.

Table 4. Causes of eosinophilia.

Allergic diseases, especially hypersensitivity of the atopic type

(e.g. bronchial asthma, hay fever, urticaria and food sensitivity)

Parasitic diseases (e.g. amoebiasis, hookworm, ascariasis, tapeworm infestation, filariasis, schistosomiasis and trichinosis)

Recovery from acute infection

Certain skin diseases (e.g. psoriasis, pemphigus and dermatitis herpetiformis, urticaria and angioedema, atopic dermatitis)

Drug sensitivity

Polyarteritis nodosa, vasculitis, serum sickness

Graft-versus-host disease

Hodgkin lymphoma and some other tumors, especially clonal T-cell disorders

Metastatic malignancy with tumour necrosis

Hypereosinophilic syndrome

Chronic eosinophilic leukemia

Myeloproliferative including systemic mastocytosis

Pulmonary syndromes Eosinophilic pneumonia, transient pulmonary infiltrates (Loeffler's syndrome), Eosinophilic Granulomatosis with Polyangiitis (EGPA) and tropical pulmonary eosinophilia.

and Ancylostoma duodenale, mostly N. americanus accounting for the predominant infections. Infections are transmitted through contact with contaminated soil, infection may also be a result of drinking contaminated water, as it will introduce the hookworm larvae; causing a local pruritic, erythematous, and papu- 
lar rash. Since in this patient clear history of infection cannot be attributed to which date or incident; it was presumed to be infection in The Philippines since the larvae infection is rarely seen in Kuwait.

Hypocomplementemia is decreased plasma C3, C4 or CH50 (third component of the complement system, fourth component of the complement system and hemolytic complement activity, respectively) [10]. The presence of hypocomplementemia points to a certain differential diagnosis which includes Infectious, Renal, Rheumatological and others. Infectious causes include bacterial infections such as streptococcus infection or gram negative bacteremia; viruses like Human Immunodeficiency Virus (HIV), Hepatitis B Virus, and Hepatitis C specially with Cryoglobulinemia. Other infections such as parasitic infections are a known cause. Renal causes including Membranoproliferative glomerulonephritis and Post-Infectious Glomerulonephritis. Rheumatological causes such as SLE, Cryoglobulinemia, Vasculitis (Polyarteritis Nodosa, Urticarial Vasculitis), and rarely Rheumatoid vasculitis. This patient is most likely to have the hypocomplementemia in the context of her helminthic infection.

\section{Conflicts of Interest}

The authors declare no conflicts of interest regarding the publication of this paper.

\section{References}

[1] Gonzalez-Mayda, C. and Ranganathan, P. (2021) The Washington Manual Rheumatology Subspecialty Consult. 3rd Edition, Lippincott Williams and Wilkins, Philadelphia.

[2] Schrier, R.W. (2008) The Internal Medicine Case Book: Real Patient, Real Answer. 3rd Edition, Lippincott Williams and Wilkins, Philadelphia.

[3] Allanore, Y., Simms, R., et al. (2015) Systemic Sclerosis. Nature Reviews Disease Primers, 1, Article No. 15002. https://doi.org/10.1038/nrdp.2015.2

[4] LeRoy, E.C. (1981) Systemic Sclerosis. In: Kelley, W.N., Jr. Harris, E.D., Ruddy, S., Sledge, C.B., Eds., Textbook of Rheutautology, WB Saunders, Philadelphia.

[5] Seibold, J.R. (2005) Scleroderma Textbook of Rheumatology. Zurich Open Respository and Archive, University of Zurich.

[6] Denton, C.P., Hughes, M., Gak, N., Vila, J., Buch, M.H., et al. (2016) BSR and BHPR Guideline for the Treatment of Systemic Sclerosis. Rheumatology, 55, 1906-1910. https://doi.org/10.1093/rheumatology/kew224

[7] Hoffbrand, A.V. and Moss, P.A.H. (2014) Essential Haematology. 6th Edition, Blackwell Publishing Ltd., Oxford.

[8] Centers for Disease Control and Prevention-About Parasites (2018)

[9] Hotez, P.J., Bethony, J., Bottazzi, M.E., Brooker, S. and Buss, P. (2005) Hookworm: “The Great Infection of Mankind". PLoS Medicine, 2, e67. https://doi.org/10.1371/journal.pmed.0020067

[10] Hebert, L.A., et al. (1991) Diagnostic Significance of Hypocomplementemia. Kidney International, 39, 811-821. https://doi.org/10.1038/ki.1991.102 